\title{
BLOCK MULTIDIMENSIONAL MPSK MODULATION CODES WITH UNEQUAL ERROR PROTECTION ${ }^{1}$
}

\author{
Cristiana S. C. M. de Jesus ${ }^{2} \quad$ Jaime Portugheis \\ DECOM - FEEC - UNICAMP \\ 13081-970 Campinas, SP, Brasil \\ e-mail: jaime@decom.fee.unicamp.br
}

\begin{abstract}
Resumo: O artigo descreve a construção de códigos de bloco de modulação M-PSK multidimensional com proteção desigual de erros. Um perfil de proteção desigual para o canal com desvanecimento de Rayleigh é obtido através da partição de constelações 4-dimensionais que são o produto Cartesiano de modulações 8-PSK nãouniformes ou são o produto de modulações uniformes. Códigos novos são construídos através da técnica de codificação multinível utilizando códigos componentes binários extremamente simples. Os códigos construídos são adequados para o codificador de voz VSELP descrito no sistema móvel IS-54. Um dos códigos construídos possui quatro níveis de proteção $\mathrm{e}$ proporciona um ganho de codificação de 16,0 dB (para uma SEGSNR de 2,0 dB) quando comparado a um sistema que utiliza modulação 4-PSK não codificada.
\end{abstract}

\begin{abstract}
The construction of block multidimensional M-PSK modulation codes with unequal error protection (UEP) is considered. An UEP profile for the Rayleigh fading channel is obtained by partitioning fourdimensional constellations that are Cartesian products of non-uniform 8-PSK modulations or are subsets of a product of uniform modulations. New codes are constructed by using the multilevel coding technique with very simple binary component codes. These codes are matched to the vector-sum excited linear predictive (VSELP) codec described in the IS-54 mobile system. One of the constructed codes has four levels of protection and achieves a coding gain of $16.0 \mathrm{~dB}$ (at a SEGSNR of $2.0 \mathrm{~dB}$ ) in comparison with a system that employs uncoded 4-PSK modulation.
\end{abstract}

Keywords: Multidimensional M-PSK modulation, multilevel codes, unequal error protection

\section{INTRODUCTION}

The combination of channel coding and modulation (coded modulation) is essential for the design of power and bandwidth efficient digital transmission schemes. On Gaussian channels, the minimum squared Euclidean distance of the code is the parameter that plays the central role as a design criterion. On fading channels, not only the minimum squared Euclidean distance, but also the minimum Hamming distance and the minimum product distance of the code should be considered in the design of the codes[1].

Nowadays in cellular mobile radio systems, the speech coders have coded bits with different sensibilities to channel errors. Therefore, it is necessary to have channel coding with unequal error protection capabilities to make the best use of channel redundancy.

A desirable feature of a technique for constructing unequal error protection codes is the possibility to control the data rates, the fraction of data that is deemed to be more important and the unequal error probability profile. Although the use of multilevel constructions with twodimensional modulations the above mentioned parameters can be controlled in a flexible manner [2], the use of multidimensional modulations offers more advantages. It not only guarantees flexibility on controlling those parameters but also opens many possibilities of applying multistage decoding algorithms [3].

In this paper we propose new block multidimensional MPSK modulation codes with UEP for the Rayleigh fading channel. The news codes are constructed using the multilevel coding technique with simple binary component codes and uniform and non-uniform multidimensional M-PSK modulations. We are looking for codes matched to the $7.95 \mathrm{kbit} / \mathrm{s}$ VSELP codec described in the IS-54 mobile system [9]. The VSELP codec has a frame of 159 bits. The error sensitivity of each bit was obtained by the method described in [4].

The paper is organised as follows. In section 2 the bit error sensitivity analysis of the VSELP codec is presented. In Section 3 the channel model is described and some considerations about the performance parameters are done. In Section 4 the construction of multidimensional modulation based on uniform and non-uniform 2dimensional constellations is described. Section 5 shows

\footnotetext{
${ }^{1}$ Parts of this paper were accepted for presentation at DSPCS' 96, Perth, Australia, September, 96.

${ }^{2}$ Now with Ericsson Telecomunicações S.A., São Paulo ,Brazil.
} 
some new codes and Section 6 their performance. Finally, in Section 7 some conclusions are drawn.

\section{VSELP BIT ERROR SENSITIVITY ANALYSIS}

Let the original speech signal, $x(t)$, be divided into $J$ non-overlapping segments of duration $T_{B}$. Let $q(t)$ be the reconstructed signal when no errors have occurred. Let $q_{i}(t)$ be the reconstructed signal when a single bit error has occurred in position $i$ of every segment. The segmental signal-to-noise ratio (SEGSNR) can be approximated as [4]:

$$
\operatorname{EGSSNR}=-(1 / J) \sum_{\mathrm{j}=1}^{\mathrm{J}} 10 \log \left[Q^{j}+\sum_{i=1}^{159} P_{i} A_{i}^{j}\right],
$$

where $P_{i}$ is the bit error probability in position $i$,

$$
Q^{j}=\int_{(j-1) T_{B}}^{j T_{B}}[x(t)-q(t)]^{2} d t / \int_{(j-1) T_{B}}^{j T_{B}}[x(t)]^{2} d t
$$

is the quantization noise normalised with the speech signal power, and

$$
A_{i}^{j}=\int_{(j-1) T_{B}}^{j T_{B}}\left[x(t)-q_{i}(t)\right]^{2} d t / \int_{(j-1) T_{B}}^{j T_{B}}[x(t)]^{2} d t
$$

By setting $Q^{j}$ to zero (for all $j$ ), $P_{i}=1$ and $P_{l}=0, l \neq i$, the SEGSNR is then reduced to $-(1 / J) \sum_{\mathrm{j}=1}^{\mathrm{J}} 10 \log A_{i}^{j}$. This expression was defined in [4] as the sensitivity of the bit in position $i$ (the average normalised noise power caused by an error in position $i$ ). Figure 1 shows the values of this sensitivity for each of the 159 bits. It can be seen that two levels of protection implies an amount of $50 \%$ of important data.

\section{CHANNEL MODEL}

The Rayleigh fading channel can be described as

$$
\mathrm{r}_{\mathrm{k}}=\rho \cdot \mathrm{x}_{\mathrm{k}}+\mathrm{n}_{\mathrm{k}}
$$

where $x_{k}$ is the kth 2-dimensional symbol transmitted, $r_{k}$ is the kth symbol received and $n_{k}$ is the white Gaussian noise with zero mean and variance $N_{0} / 2$. The fading amplitude $\rho$ has Rayleigh density given by

$$
p(\rho)= \begin{cases}2 \rho \exp \left\{-\rho^{2}\right\} ; & \rho \geq 0 \\ 0 ; & \rho<0\end{cases}
$$

where the average energy per received symbol is $\mathrm{E}\left\{\rho^{2} E_{m}\right\}=E_{m}$. Therefore, the signal to noise ratio $\left(E_{b} / N_{0}\right)$ is given by $E_{b} / N_{0}=E_{m} /\left(\right.$ R. $\left.N_{0}\right)$, where $E_{b}$ is the average energy per information bit and $\mathrm{R}$ is the bits per symbol rate. For our constructions we assumed a memoryless channel, achieved through a perfect interleaving, perfect channel state information (CSI), ideal coherent detection and a Gaussian decoding metric.

From these assumptions, it can be seen that the pairwise error probability between sequences $X$ and $\hat{X}$ can be approximated by

$$
P(X \rightarrow \hat{X}) \cong \frac{1}{d_{p}(X, \hat{X})}\left(\frac{4}{E_{m} / N_{0}}\right)^{d_{H}(x, \hat{x})}
$$

where $E_{m}$ is average energy per transmitted symbol, $d_{H}(X, \hat{X})$ is the Hamming distance between the sequences $X$ e $\hat{X}$, and ${ }_{d_{p}}(X, \hat{X})$ is the product distance defined as

$$
d_{p}(X, \hat{X})=\prod_{n \in \eta}\left|x_{n}-\hat{x}_{n}\right|^{2}
$$

where $\eta$ is the set of all $n$ that $x_{n} \neq \hat{x}_{n}$.

In equation (2) we can notice that the Hamming distance $d_{H}(X, \hat{X})$ and the product distance $d_{p}(X, \hat{X})$ are important for the code performance. It is worthy of mention that similar results can be obtained for other channel models (no CSI, M-DPSK signals, Ricean channels), if the values of channel SNR are sufficiently high $[1,5]$.

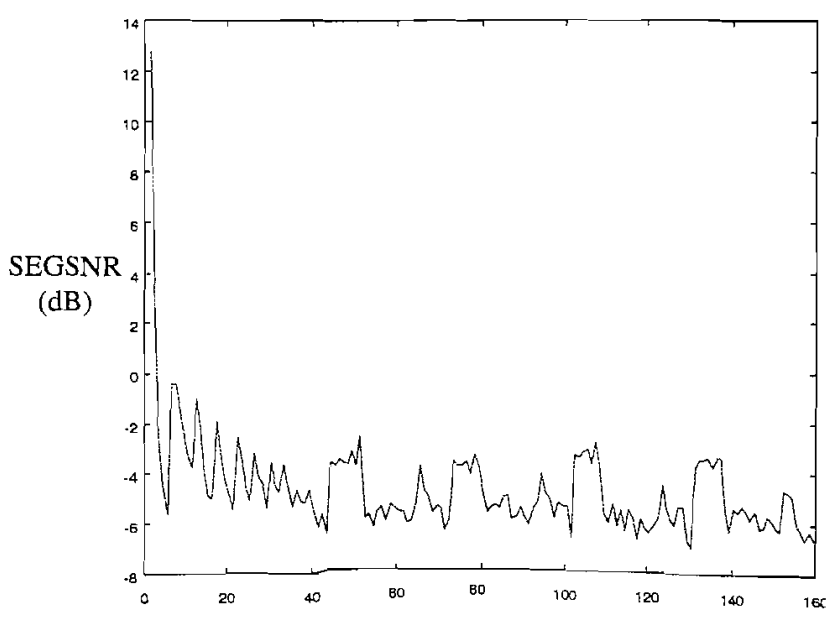

Fig.1 - Bit error sensitivity of VSELP coded bits. 


\section{MULTILEVEL CONSTRUCTIONS AND MULTIDIMENSIONAL CONSTELLATIONS}

Consider an initial constellation $\mathrm{S}$ with $2^{\mathrm{L}}$ signals. We will define a sequence of $\mathrm{L}$ binary partitions. Each subset of the ( $l-1)$-th partition contains two subsets of the $l$-th partition and has $2^{(\mathrm{L}-l+1)}$ signals. We will label with the numbers 0 and 1 the two subsets at level 1 corresponding to the subsets at level $(1-1)$. Therefore, the binary vector with $\mathrm{L}$ positions $\left(\mathrm{c}_{1}, \mathrm{c}_{2}, \ldots, \mathrm{c}_{\mathrm{L}}\right)$ can be associated to a specific signal s from $S, \quad s=f\left(c_{1}, c_{2}, \ldots, c_{L}\right)[5]$.

We will be considering multidimensional constellations $S$ which are the Cartesian product of 2-dimensional M-PSK modulations. Thus, a signal $\mathrm{s}$ of $\mathrm{S}$ is a sequence of $n$ PSK signals. We will define the minimum Hamming distance, $\delta_{H}^{l}$, as the shortest distance among the minimum distances of the subsets at level 1 of the partition. And associate to each level a minimum product distance, $\delta_{P}^{l}$, which is the shortest distance (between) the signals that have minimum Hamming distance $\delta_{H}^{l}$.

Figure 2 describes a multilevel construction. Initially, we have $\mathrm{L}$ encoders corresponding to the binary codes $\mathrm{C}_{1}, \mathrm{C}_{2}, \ldots \mathrm{C}_{\mathrm{L}}$, where $\mathrm{C}_{\mathrm{l}}=\left(\mathrm{N}, \mathrm{K}^{l}, \mathrm{~d}_{\mathrm{H}}{ }^{l}\right)$ is a code with length $\mathrm{N}$, $\mathrm{K}^{l}$ information bits and minimum Hamming distance $\mathrm{d}_{\mathrm{H}}{ }^{l}$.

Constructing the LxN matrix,

$$
V=\left[\begin{array}{cccc}
c_{11} & c_{12} & \cdots & c_{1 N} \\
c_{21} & c_{22} & \cdots & c_{2 N} \\
\vdots & \vdots & & \vdots \\
c_{L 1} & c_{L 2} & \cdots & c_{L N}
\end{array}\right]
$$

where the lth row of $\mathrm{V}$ is a codeword from $\mathrm{C}_{l}$, and replacing each column from $\mathrm{V}$ by a signal from $\mathrm{S}$ defined by the partitions

$$
s_{k}=f\left(c_{1 k}, c_{2 k}, \ldots c_{L k}\right)
$$

we obtain a sequence $\left(s_{1}, s_{2}, \ldots s_{N}\right)$ from the multilevel code.

The multilevel code rate in information bits per PSK

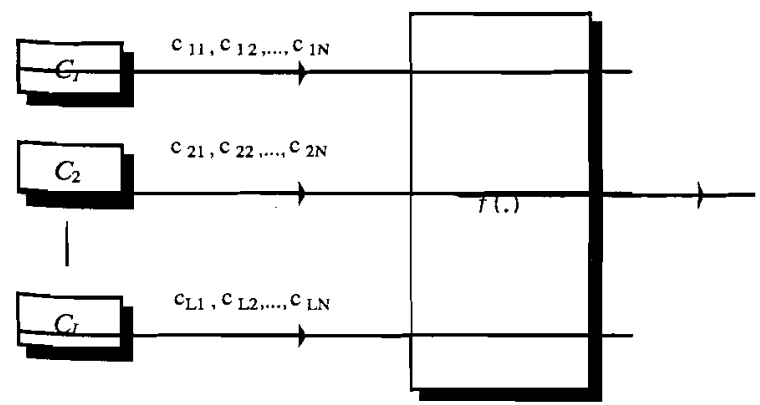

Fig.2-Scheme of a multilevel coding system symbol is then

$$
R=\sum_{l=1}^{L} K_{l} / n \cdot N
$$

The minimum Hamming distance of the resultant code at each level, $\left(d_{\mathrm{H}}^{l}\right)_{\mathrm{res}}$, is bounded by the product of the component code minimum Hamming distance and the minimum Hamming distance of that partition level, $\left(d_{\mathrm{H}}^{l}\right.$ )$_{\text {res }} \geq \delta_{H}^{l} \cdot \mathrm{d}_{\mathrm{H}}{ }^{l}$. And the resultant code product distance for the $l$ th, $d_{\mathrm{P}}^{l}$ is given by $d_{\mathrm{P}}^{l} \geq\left(\delta_{P}^{l}\right)^{d_{H}^{l}}[6]$.

In this paper we use multidimensional constellations as initial constellation, the Cartesian products of 2dimensional 8-PSK modulations, that is, $n=2$. We have 2-dimensional non-uniform constellations, $S_{1}$ e $S_{2}$ as shown in Figure 3. The third one $S_{3}$ is a uniform constellation, but the total set of signals of the multidimensional constellation was reduced for only 32 signals $(8 \times 8)$ - PSK to achieve UEP properties as in the case of non-uniform constellations.

Usually in multilevel constructions, the minimum squared Euclidean distance between two signals increases successively in each level of the partitions. In this case, however, as we need to have unequal error protection, and we will make multistage decoding, it is interesting to have great distances in the first levels whenever possible. To illustrate this fact we will show the first levels of the partition made in the constellation $\left(S_{3}\right)^{2}$, with the distances $\delta_{P}^{l}$ e $\delta_{H}^{l}$ in each level. We initially have a set with 32 signals.

$$
\Omega=\left[\begin{array}{llllllll}
17 & 27 & 31 & 41 & 57 & 67 & 71 & 01 \\
10 & 20 & 32 & 42 & 50 & 60 & 72 & 02 \\
13 & 23 & 35 & 45 & 53 & 63 & 75 & 05 \\
14 & 24 & 36 & 46 & 54 & 64 & 76 & 06
\end{array}\right]
$$

In the first level, the minimum Hamming distance $\delta_{H}^{1}$ is equal to 1 , and the minimum product distance $\delta_{P}^{1}$ is 3,41 . The two subsets are shown below.

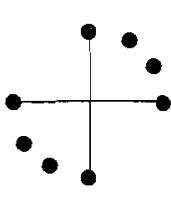

S1

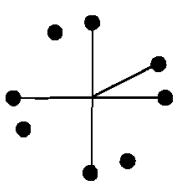

S2

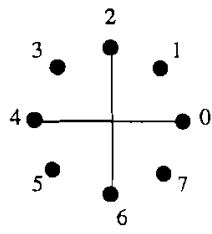

S3
Fig. 3 - Signal constellations $S_{1}, S_{2}, S_{3}$. 


$\Omega_{0}=\left[\begin{array}{llll}17 & 27 & 31 & 41 \\ 10 & 20 & 32 & 42 \\ 13 & 23 & 35 & 45 \\ 14 & 24 & 36 & 46\end{array}\right]$
$\Omega_{1}=\left[\begin{array}{llll}57 & 67 & 71 & 01 \\ 50 & 60 & 72 & 02 \\ 53 & 63 & 75 & 05 \\ 54 & 64 & 76 & 06\end{array}\right]$

In the second level the minimum Hamming distance between the signals is equal to 1 again, and the minimum product distance 3,41. The four subsets are shown below respectively.

$$
\begin{aligned}
\Omega_{00} & =\left[\begin{array}{llll}
17 & 27 & 31 & 41 \\
10 & 20 & 32 & 42
\end{array}\right] \\
\Omega_{01} & =\left[\begin{array}{llll}
13 & 23 & 35 & 45 \\
14 & 24 & 36 & 46
\end{array}\right] \\
\Omega_{10} & =\left[\begin{array}{llll}
57 & 67 & 71 & 01 \\
50 & 60 & 72 & 02
\end{array}\right] \\
\Omega_{11} & =\left[\begin{array}{llll}
53 & 63 & 75 & 05 \\
54 & 64 & 76 & 06
\end{array}\right]
\end{aligned}
$$

In the next three levels we will have respectively the Hamming distances 2,1 and 2. And the product distances $2,(0,58)$ and $\left(0,58^{2}\right)$.

The partitions of constellations $\left(S_{1}\right)^{2}$ and $\left(S_{2}\right)^{2}$ can be described by using Cartesian products of subsets of $S_{1}$ and $S_{2}$, respectively. In the following, we show these products for levels 1,2 and 3 of the partition.

\section{Partition of $\left(S_{1}\right)^{2}$}

Leve!

$$
\begin{array}{ll}
1 \quad \Omega_{0}=\{0,1,2,3\} \times\{0,1, \ldots, 7\}, & \Omega_{1}=\{4,5,6,7\} \times\{0,1, \ldots, 7\} \\
2 \quad \Omega_{00}=\{0,1,2,3\} \times\{0,1,2,3\}, & \Omega_{01}=\{0,1,2,3\} \times\{4,5,6,7\} \\
\Omega_{10}=\{4,5,6,7\} \times\{0,1,2,3\}, & \Omega_{11}=\{4,5,6,7\} \times\{4,5,6,7\} \\
3 \quad \Omega_{000}=\{0,2\} \times\{0,1,2,3\}, & \Omega_{001}=\{1,3\} \times\{0,1,2,3\} \\
\Omega_{010}=\{0,2\} \times\{4,5,6,7\}, & \Omega_{011}=\{1,3\} \times\{4,5,6,7\} \\
\Omega_{100}=\{4,6\} \times\{0,1,2,3\}, & \Omega_{101}=\{5,7\} \times\{0,1,2,3\} \\
\Omega_{110}=\{4,6\} \times\{4,5,6,7\}, & \Omega_{111}=\{5,7\} \times\{4,5,6,7\}
\end{array}
$$

Partition of $\left(S_{2}\right)^{2}$

$$
\begin{aligned}
& \text { Level } \\
& 1 \quad \Omega_{0}=\{0,1,4,5\} \times\{0,1, \ldots, 7\}, \Omega_{1}=\{2,3,6,7\} \times\{0,1, \ldots, 7\} \\
& 2 \Omega_{00}=\{0,1,4,5\} \times\{0,1,4,5\}, \Omega_{01}=\{0,1,4,5\} \times\{2,3,6,7\} \\
& \Omega_{10}=\{2,3,6,7\} \times\{0,1,4,5\}, \Omega_{11}=\{2,3,6,7\} \times\{2,3,6,7\} \\
& 3 \quad \Omega_{000}=\{0,4\} \times\{0,1,4,5\}, \quad \Omega_{001}=\{1,5\} \times\{0,1,4,5\} \\
& \Omega_{010}=\{0,4\} \times\{2,3,6,7\}, \quad \Omega_{011}=\{1,5\} \times\{2,3,6,7\} \\
& \Omega_{100}=\{2,6\} \times\{0,1,4,5\}, \quad \Omega_{101}=\{3,7\} \times\{0,1,4,5\} \\
& \Omega_{110}=\{2,6\} \times\{2,3,6,7\}, \quad \Omega_{111}=\{3,7\} \times\{2,3,6,7\}
\end{aligned}
$$

The multistage decoding method used is a sub optimum method, which has an excellent trade off between error probability and implementation complexity [7,1]. The idea of multistage decoding is to decode the received word $\vec{R}=\left(\vec{r}_{1}, \vec{r}_{2}, \cdots, \vec{r}_{N}\right)$ in L stages passing the codeword of code $C_{i}$ estimated in the $i$-th stage, $i=1,2, \ldots, L$ -1 , to the subsequent stages. At the first stage, for each received vector $\vec{r}_{k}, \mathrm{k}=1,2, \ldots, \mathrm{N}$, the decoder obtains the nearest multidimensional M-PSK signals in subsets $\Omega_{0}, \Omega_{1}$ and their squared Euclidean distances $d^{2}\left(\vec{r}_{k}, s\left(\Omega_{0}\right)\right), d^{2}\left(\vec{r}_{k}, s\left(\Omega_{1}\right)\right)$. Based on this information, the decoder of $C_{1}$ chooses the codeword $\vec{c}_{1}=\left(c_{11}, c_{12}, \ldots, c_{1 N}\right) \quad$ which minimizes $\sum_{k=1}^{N} d^{2}\left(\vec{r}_{k}, s\left(\Omega_{c_{1 k}}\right)\right)$. This codeword is passed to the subsequent decoders. At the second stage, for each received vector $\vec{r}_{k}, \mathrm{k}=1,2, \ldots, \mathrm{N}$, the decoder of $\mathrm{C}_{2}$ obtains the nearest multidimensional M-PSK signals in subsets $\Omega_{c_{1 k}, 0}, \Omega_{c_{1 k}, 1}$ and their squared Euclidean distances $\quad d^{2}\left(\vec{r}_{k}, s\left(\Omega_{c_{1 k}, 0}\right)\right), d^{2}\left(\vec{r}_{k}, s\left(\Omega_{c_{1 k}, 1}\right)\right)$. Based on this information, the decoder of $\mathrm{C}_{2}$ chooses the codeword $\vec{c}_{2}=\left(c_{21}, c_{22}, \ldots, c_{2 N}\right)$ which minimizes $\sum_{k=1}^{N} d^{2}\left(\vec{r}_{k}, s\left(\Omega_{c_{1 k}, c_{2 k}}\right)\right)$. This codeword is passed to the subsequent decoders. This process is repeated in all subsequent stages always using the estimates of previous stages until the codeword $\vec{c}_{L}=\left(c_{L 1}, c_{L 2}, \ldots, c_{L N}\right)$ is estimated and the multistage decoding is complete.

The above described partitions of constellations $\left(S_{1}\right)^{2}$ and $\left(S_{2}\right)^{2}$ simplify the multistage decoding. The nearest multidimensional M-PSK signals in subsets $\Omega_{0}, \quad \Omega_{1}$ and their squared Euclidean distances $d^{2}\left(\vec{r}_{k}, s\left(\Omega_{0}\right)\right), \quad d^{2}\left(\vec{r}_{k}, s\left(\Omega_{1}\right)\right)$ can be calculated based solely on the nearest M-PSK signals and their distances in the two-dimensional subsets $\{0,1,2,3\},\{4,5,6,7\},\{0,1, \ldots, 7\} \quad$ for $\quad\left(S_{1}\right)^{2} \quad$ and $\{0,1,4,5\},\{2,3,6,7\},\{0,1, \ldots, 7\}$ for $\left(S_{2}\right)^{2}$. A similar 


\begin{tabular}{|c|c|c|c|c|c|c|}
\hline Level & \multicolumn{2}{|c|}{$B_{1}$} & \multicolumn{2}{c|}{$B_{2}$} & \multicolumn{2}{c|}{$B_{3}$} \\
\hline$l$ & $\left(\mathrm{~d}_{\mathrm{H}}^{l}\right)_{\text {res }}$ & $\mathrm{d}_{\mathrm{p}}^{l}$ & $\left(\mathrm{~d}_{\mathrm{H})_{\text {res }}}\right.$ & $\mathrm{d}_{\mathrm{p}}^{l}$ & $\left(\mathrm{~d}_{\mathrm{H}}^{l}\right)_{\text {res }}$ & $\mathrm{d}_{\mathrm{p}}^{l}$ \\
\hline 1 & 2 & 4 & 4 & 1 & 4 & $3.41^{4}$ \\
\hline 2 & 2 & 4 & 4 & 1 & 4 & $3.41^{4}$ \\
\hline 3 & 1 & 0.26 & 4 & $3.73^{4}$ & $2 \times 1$ & 2 \\
\hline 4 & 1 & 0.26 & 4 & $3.73^{4}$ & 1 & 0.58 \\
\hline 5 & 1 & 1 & 1 & 0.26 & $2 \times 1$ & $0.58^{2}$ \\
\hline 6 & 1 & 1 & 1 & 0.26 & - & - \\
\hline
\end{tabular}

Table 1.

procedure can be applied to compute the nearest signals and their squared distances for the second stage. At the third stage and subsequent stages we need additionally the nearest M-PSK signals and their distances in the twodimensional subsets $\{0,2\},\{1,3\},\{4,6\},\{5,7\}$ for $\left(S_{1}\right)^{2}$ and $\{0,4\},\{1,5\},\{2,6\},\{3,7\}$ for $\left(S_{2}\right)^{2}$.

\section{EXAMPLES}

We present some examples of construction.

Example1: We consider a binary partition of the 4dimensional signal constellation $\left(S_{1}\right)^{2}$. The first and the second levels of the partition are encoded by the code $C_{1}=C_{2}=(16,15,2)$. We left the other levels uncoded, i.e. $C_{3}=C_{4}=C_{5}=C_{6}=(16,16,1)$. The rate of the multilevel code $B_{1}$ is then 2.94 bits/symbol. The distance profile of $B_{1}$ is given in Table 1 . From the table we expect three different levels of protection: the information bits of $C_{1}$ and $C_{2}$ should have lower error probability than those of $C_{5}$ and $C_{6} . C_{3}$ and $C_{4}$ should have worse performance than $C_{5}$ and $C_{6}$. The information bits of $C_{1}$ and $C_{2}$ represent an amount of $30 \%$ of important data.

Example 2: We consider a binary partition of the 4dimensional signal constellation $\left(S_{2}\right)^{2}$. The first and the second levels of the partition are encoded by the code $C_{1}=C_{2}=C_{3}=C_{4}=(8,4,4)$. We left the others levels uncoded. The rate of the multilevel code $B_{2}$ is then 2.0 bits/symbol. The distance profile of $B_{2}$ is given in Table 1 . From the table we expect three different levels of protection: the information bits of $C_{3}$ and $C_{4}$ should have a slightly lower error probability than those of $C_{1}$ e $C_{2}$ and much lower than the uncoded bits of the levels 5 and 6 .
The information bits of $C_{3}, C_{4}, C_{1}$ and $C_{2}$ represent an amount of $50 \%$ of important data.

Example 3: In this example we consider a binary partition of a subset of the 4-dimensional signal constellation $\left(S_{3}\right)^{2}$ with only 32 signals. The first and the second levels of the partition are encoded by the code $C_{1}=C_{2}=(8,4,4)$. We left the others levels uncoded. The rate of multilevel code $B_{3}$ is then 2.0 bits/symbol. The distance profile of $B_{3}$ is given in Table 1. The information bits of $C_{3}, C_{1}$ and $C_{2}$ represent an amount of $50 \%$ of important data.

\section{SIMULATION RESULTS}

We have simulated the transmission of blocks of 159 information bits using encoders for the multilevel codes $B_{2}$ and $B_{3}$ at the transmitter and standard multistage decoding at the receiver. For code $B_{1}$, blocks of 94 information bits were used. The coding gains are considered over uncoded 4-PSK and to a bit error probability of $10^{-3}$.

Figure 4 shows simulations results for code $B_{1}$. The figure shows the expected three levels of protection. The coding gains are about $9 \mathrm{~dB},-4 \mathrm{~dB}$ and $-6 \mathrm{~dB}$ for the first, the second and the third level, respectively.

Figure 5 shows simulations results for code $B_{2}$. The figure shows the expected three levels of protection. The coding gains are about $16 \mathrm{~dB}, 15 \mathrm{~dB}$ and $-8 \mathrm{~dB}$ for the first, the second and the third level, respectively.

Figure 6 shows simulation results for code $B_{3}$. The figure shows four levels of protection: the information bits of the binary component codes $C_{1}, C_{2}$ form the first level, the information bits of $C_{3}$ the second level, of $C_{5}$ the third level and of $C_{4}$ the fourth level. The coding gains are about $19 \mathrm{~dB}, 12 \mathrm{~dB},-4 \mathrm{~dB}$ e $-8 \mathrm{~dB}$ for the first, the second, the third and the fourth level, respectively.

It is possible to modify the codes of the above examples to obtain some gain for the less important data while sacrificing the data rate of the multilevel code or some of the gain of the most important data.

The overall performances of the coded systems has been evaluated by using the speech SEGSNR (defined in Section 2) as an objective quality measure $[4,8]$. Figure 7 shows the SEGSNR (as a function of $E_{b} / N_{0}$ ) for three different systems. Curves $C S_{3}, C S_{2}$ and $C S_{1}$ show the behaviour of the VSELP codec together with code $B_{3}$, code $B_{2}$ and uncoded 4-PSK modulation, respectively. At a speech SEGSNR of $2.0 \mathrm{~dB}$ the coding gain of system $C S_{2}$ over $C S_{3}$ is about $14.0 \mathrm{~dB}$. System $C S_{1}$ achieves an additional gain of about $2.0 \mathrm{~dB}$. 


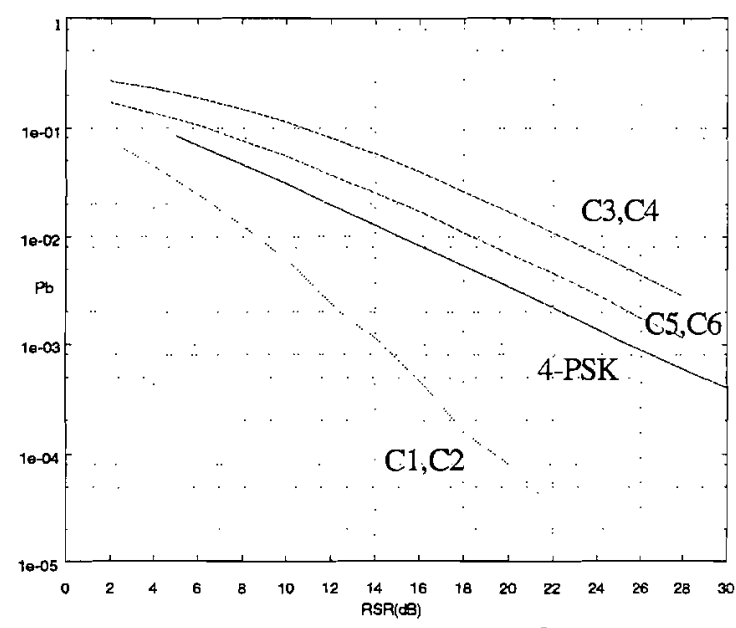

Fig.4 - Performance of code $B 1$

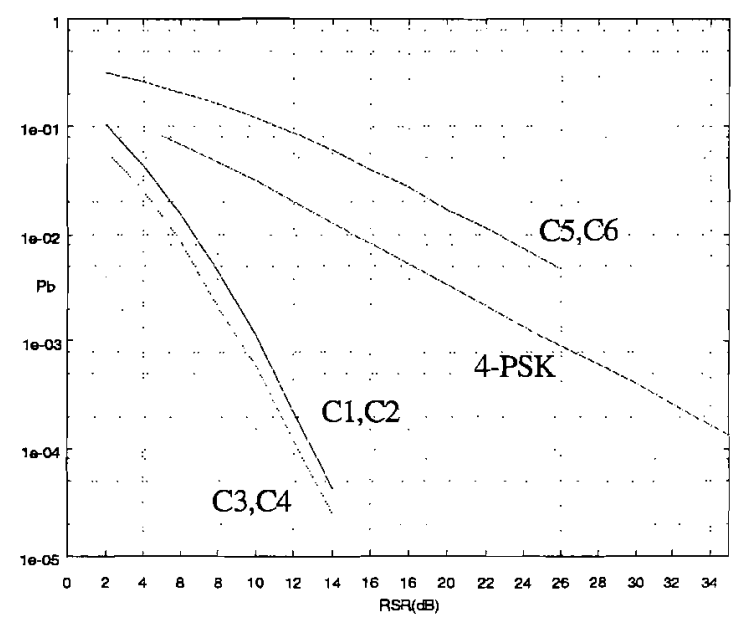

Fig.5 - Performance of code $B_{2}$

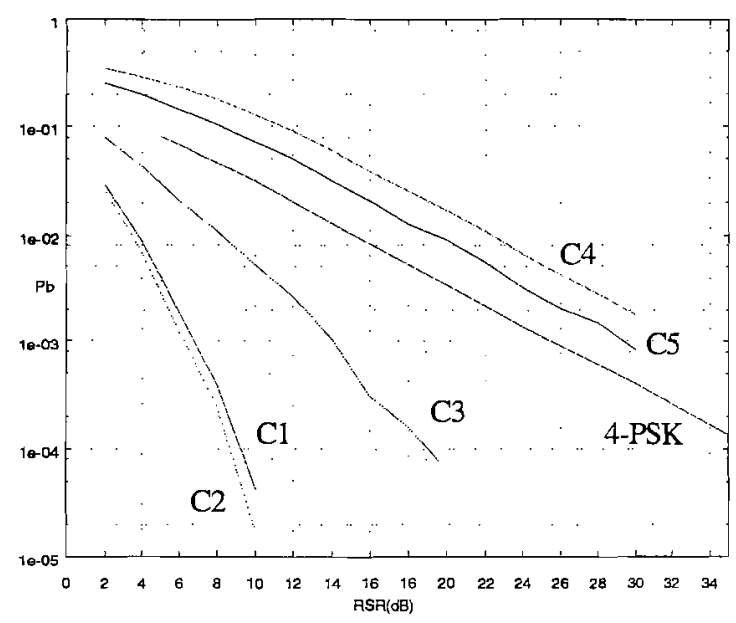

Fig.6 - Performance of code $\mathrm{B}_{3}$

\section{CONCLUSIONS}

We have described the construction of new multilevel block codes with UEP based on uniform and non-uniform 4-dimentional 8-PSK modulations. One of the constructed codes (code $B_{3}$ ) has four levels of UEP and when used together with a VSELP codec achieves a coding gain of $16.0 \mathrm{~dB}$ (at a SEGSNR of $2.0 \mathrm{~dB}$ ) in comparison with a system that employs uncoded 4-PSK modulation. It is important to notice that this gain is obtained although the less important data is under the effect of a bit error probability worse than that of uncoded transmission.

\section{Acknowledgments}

The authors would like to thank Professor Fabio Violaro for guidance and helpful discussions and Eliana De Martino who kindly supplied the VSELP simulation program. This work has been partially supported by CNPq-Conselho Nacional de Pesquisa under Grant $301045 / 92-5$.

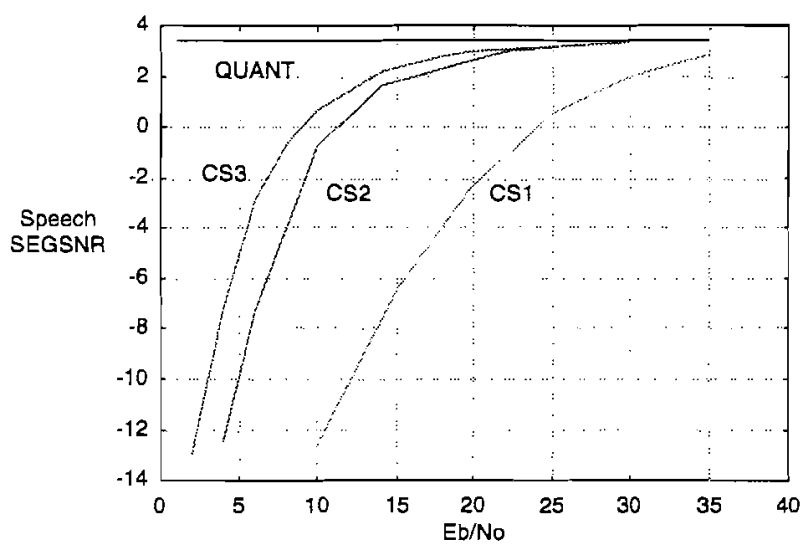

Fig. 7 - Speech SEGSNR versus Channel SNR

\section{REFERENCES}

[1]N.Seshadri, C.-E. W. Sundberg, "Multilevel Trellis Coded Modulations for the Rayleigh Fading Channel", IEEE Trans. Commun., vol.41,pp.13001310, Sept. 1993.

[2]N.Seshadri, C.-E. W. Sundberg, "Multilevel Block Coded Modulations with unequal error protection for the Rayleigh Fading Channel", ETT, Vol.4, pp.325-334, May-June,1993.

[3]J.Portugheis, "On block multidimensional M-PSK modulation codes", Procedings 14th Symp. Inform. Theory and Applications, Ibusuki, Japan, pp. 337380, Dec. 1991.

[4]R.V. Cox, J.Hagenauer, N.Seshadri, C.-E. W. Sundberg, "Subband Speech Coding and Matched Convolutional Channel Coding for Mobile Radio Channels", IEEE Trans. on Signal Processing, vol. 39, pp.1717-1731, Aug.,1991.

[5]E. Biglieri, D. Divsalar, P.J.McLane, M.K. Simon, "Introduction to Trellis-Coded Modulation with Applications", Macmillan, pp. 224-226, USA, 1991.

[6]C.S.C.M. de Jesus, Master Thesis, in preparation. 
[7]H.Imai, S.Hirakawa, "A new multilevel coding method using error-correcting codes", IEEE Trans. on Inform. Theory, vol. IT-23, pp. 371-377, May 1977.

[8]Hui Li, Peter Noll "Hybrid Phase Trellis-Coded Modulation for Coding with Unequal Error Protection", Int. Symposium on Signals, Systems and Electronics, Book I, pp. 113-116, Paris, September 1-4, 1992.

[9]EIA/TIA Project Number 2215: Cellular System "Dual-Mode Mobile Station - Base Station Compatibility Standard" - IS-54 - December, 1989.

Cristiana S. C. M. de Jesus was born in Recife, Brazil, in 1971. She received a degree in electrical engineering in 1993 from the Universidade Federal de Pernambuco, Recife, Brazil. She is currently working towards the Master degree at the Universidade Estadual de Campinas, Campinas, Brazil. Since February 1996, she has also been working as a Radio Network Engineer at Ericsson Telecomunicações S.A., São Paulo, Brazil.

Her research interests include coding and mobile radio communications.

Jaime Portugheis received a degree in electrical engineering in 1983 from the Universidade Federal de Pernambuco, Recife, Brazil, a M. S. degree in communications in 1987 from Universidade Estadual de Campinas (Unicamp), Campinas, Brazil and the Dr.-Ing. degree in 1992 from Technische Hochschule Darmstadt, Darmstadt, Germany. In october 1992 he joined the Faculty of Electrical and Computing Engineering of Unicamp, where he is an Assistant Professor.

His research interests include coded modulation and multiuser communications. 